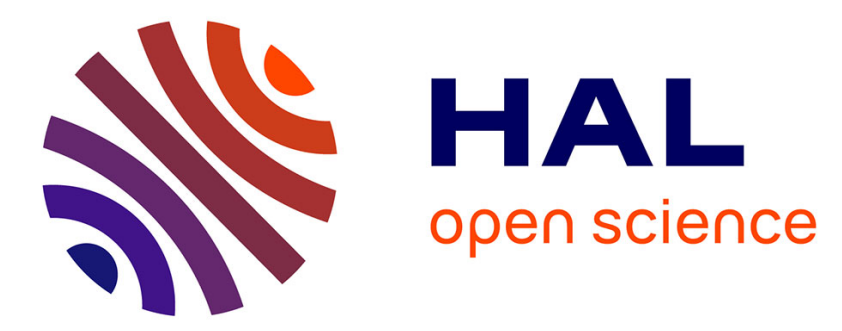

\title{
Assisting Human Balance in Standing With a Robotic Exoskeleton
}

Ildar Farkhatdinov, Julia Ebert, Gijs van Oort, Mark Vlutters, Edwin van Asseldonk, Etienne Burdet

\section{To cite this version:}

Ildar Farkhatdinov, Julia Ebert, Gijs van Oort, Mark Vlutters, Edwin van Asseldonk, et al.. Assisting Human Balance in Standing With a Robotic Exoskeleton. IEEE Robotics and Automation Letters, 2019, 4 (2), pp.414-421. 10.1109/lra.2018.2890671 . hal-02045870

\section{HAL Id: hal-02045870 https://hal.science/hal-02045870}

Submitted on 28 Apr 2019

HAL is a multi-disciplinary open access archive for the deposit and dissemination of scientific research documents, whether they are published or not. The documents may come from teaching and research institutions in France or abroad, or from public or private research centers.
L'archive ouverte pluridisciplinaire HAL, est destinée au dépôt et à la diffusion de documents scientifiques de niveau recherche, publiés ou non, émanant des établissements d'enseignement et de recherche français ou étrangers, des laboratoires publics ou privés. 


\title{
Assisting Human Balance in Standing with a Robotic Exoskeleton
}

\author{
Ildar Farkhatdinov ${ }^{1,2}$, Julia Ebert ${ }^{2,3}$, Gijs van Oort ${ }^{4}$, Mark Vlutters ${ }^{4}$ \\ Edwin van Asseldonk ${ }^{4}$ and Etienne Burdet ${ }^{2,5}$
}

\begin{abstract}
This paper presents an experimental study on balance recovery control with a lower limb exoskeleton robot. Four participants were subjected to a perturbation during standing, a forward force impulse applied to their pelvis that forced them to step forward with the right leg for balance recovery. Trials with and without exoskeleton assistance to move the stepping legs thigh were conducted to investigate the influence of the exoskeletons control assistance on balancing performance and a potential adaptation. Analysis of the body kinematics and muscle activation demonstrates that robotic assistance: (1) was easy to use and did not require learning, nor inhibited the healthy stepping behavior; (2) it modified the stepping leg trajectories by increasing hip and knee movement; (3) increased reaction speed and decreased the step duration, (4) generally increased biceps femoris and rectus femoris muscle activity.
\end{abstract}

Index Terms-Prosthetics and Exoskeletons; Physically Assistive Devices

\section{INTRODUCTION}

$\mathbf{L}$ OWER limb robotic exoskeletons have been proposed for human performance enhancement and neuromotor rehabilitation [1], [2], [3]. However, improving the safety and performance of wearable robotic systems, and the development of novel functionalities, still remains challenging research topics. Gait assistance during walking is the main application for the majority of lower limb exoskeleton robots. While some of the previous works [4], [5] state that modification of human gait characteristics improves stability in walking, very few results were published on improving balance function with the help of wearable exoskeletons. Balance control in standing and walking is a crucial exoskeleton control function, which should be considered in the development and evaluation of wearable robots for posture and gait assistance [6].

The present work describes an experimental study on robot-assisted stepping for balance recovery in case of upper body force perturbation in healthy humans. When losing balance, humans generally use a stepping strategy, which is a robust balance recovery strategy in healthy adults [7], [8], [9]. Currently there are no robotic exoskeletons which can support stepping for balance recovery, and it is unclear how an exoskeleton controller should be designed

\footnotetext{
${ }^{1}$ School of Electrical Engineering and Computer Science, Queen Mary University of London, UK i.farkhatdinov at qmul.ac.uk.

${ }^{2}$ Department of Bioengineering, Imperial College of Science, Technology and Medicine, UK e.burdet at imperial.ac.uk.

${ }^{3}$ Computer Science, Harvard University, USA.

${ }^{4}$ Department of Biomechanical Engineering, Technical Medical Centre, University of Twente, Netherlands.

${ }^{5}$ Nanyang Technological University, Singapore.
}

to act efficiently and cooperatively with its user in order to maintain stability during standing. We addressed this challenging question in more depth through the development and experimental evaluation of cooperative balance recovery control in a human-exoskeleton system. More specifically we investigated the effect of a simple stepping assistance torque impulse produced by an exoskeleton and applied to a hip joint flexion to improve balance recovery after the upperbody perturbation.

We envisioned that the exoskeleton could help stepping and thus stability recovery, because $\left(\mathrm{H}_{1}\right)$ the users would adapt to the assistance quickly (no learning would be required); $\left(\mathrm{H}_{2}\right)$ the exoskeleton could make balance recovery faster; $\left(\mathrm{H}_{3}\right)$ robotic assistance would help stepping thus reducing muscle activation. To test these three hypotheses we designed a simple balance recovery controller for a lower limb exoskeleton which can detect external body perturbations and provide assistance to the exoskeleton's user. Using the sophisticated LOPES III research exoskeleton [10], we could conduct a systematic experimental study in which participants pelvis was perturbed and balance recovery action with stepping could be compared with and without robotic stepping assistance.

This paper presents the experimental setup, defines the stepping control strategy with the exoskeleton, describes its implementation and the experimental protocol. We analyse the role of the assistance, muscle activation and kinematic re-

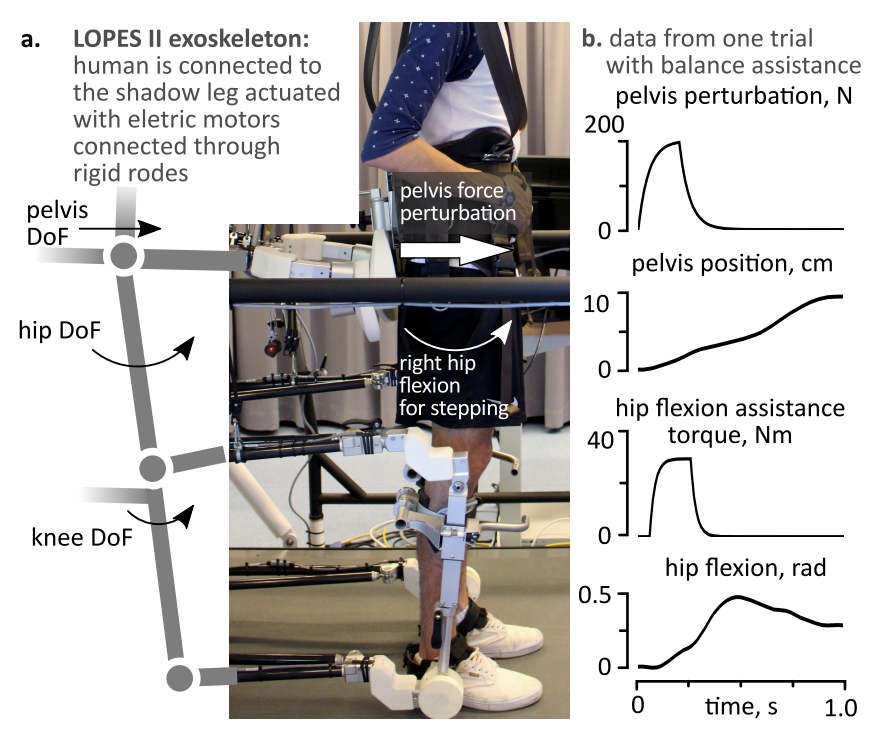

Fig. 1. a: Participant before stepping with LOPES III exoskeleton; b: Sample single trial recorded data for balance recovery assistance. 
sponse in the balance recovery performance through stepping with the robotic exoskeleton. We examine whether assistance leads to modifications of the natural stepping behavior as it is necessary to know if the exoskeleton will not disturb the healthy behavior of a user and this test can only be performed with healthy subjects. The results suggest that the simple robot-assisted stepping does not disrupt or modify the natural behavior, and enable faster reaction to destabilisation. This is a first study towards developing robot-assisted stepping to improve the stability in frail humans.

\section{EXOSKELETON BALANCE CONTROL}

State of the art. Existing works on augmenting balance with robotic exoskeletons have important limitations. In [11], [12] a lower limb exoskeleton with variable stiffness actuators was used for balance recovery, however experiments were performed with the robot only. In [13] the influence of passive exoskeleton mechanics on human biomechanics of walking and balancing was investigated. It was shown that a passive exoskeleton degraded the balancing performance when perturbations were applied to the exoskeleton's user. The importance of providing pelvis anterior movements in the exoskeleton to improve stability of walking was demonstrated in [14]. Studies [11], [14] indicated that the exoskeleton design, specifically its kinematic structure, can significantly degrade balancing function. In [15] a knee exoskeleton is used for balancing assistance based on mimicking estimated human knee joint impedance control. The experiments were conducted with one participant only and limited data was provided. A powered ankle-foot orthosis from [16] was used to improve standing balance for two spinal cord injury patients. Recently, it was shown that a robotic exoskeleton can facilitate balance recovery after unexpected slippages during walking, by applying torque to a user's hips to assist with flexion/extension [17]. The exoskeleton applied torques to a user's hips to assist with flexion/extension after slippage was detected.

Overall only a few balance-assisting controllers in lower limb robotic exoskeletons have been investigated with human-participants: balance recovery with one subject in [15] and with two subjects in [16]; influences on balance during walking with passive exoskeletons in [13], [14]. Most importantly, these studies did not consider human's active behavior in balancing with the exoskeleton [11], [12]. The study on exoskeleton-assisted balance recovery after slippage reported in [17] considered the biomechanical responses of the participants but did not analyse the related muscle activation.

Proposed balance assistance. We consider a lower limb robotic exoskeleton attached to its user at the pelvis and the legs (Fig. 1a). The general scenario considered is balance recovery from external perturbation applied at the pelvis level. In such situations, a stepping balance recovery strategy is employed depending on the magnitude of the pelvis perturbation. Direction and stepping points will depend on the direction of the perturbation, resulting in stepping with left or right leg. This stepping balance recovery action will also depend on actual human-exoskeleton posture. We propose to implement an exoskeleton balance assistance controller that will take into account the above mentioned and will assist the exoskeleton user with stepping for balance recovery once the perturbation is detected.

In the experimental study during certain trials, an assistance torque was applied after a pelvis perturbation was detected, to assist with balance recovery. The goal of the assistance was to apply external force supporting natural behavior in stepping required for balance recovery (hip flexion of the stepping leg). Because balance recovery is rapid, it is likely that the body uses simple and robust muscular control to regain balance. As such, simple torques were selected for assistance, with a filter applied to prevent rapid accelerations, which would be both unnatural and unsafe.

Perturbations were detected by pelvis anterior-posterior acceleration, $a_{p}$, and jerk, $\dot{a}_{p}$, at time $t_{k}$, crossing a threshold of $a_{\text {lim }}=2.5 \mathrm{~m} / \mathrm{s}^{2}$ :

$$
\begin{array}{r}
\text { wait for time } t_{k}: a_{p}\left(t_{k}\right)+\Delta_{p} \dot{a}_{p}\left(t_{k}\right)>a_{l i m}, \\
\tau_{\circ}=\gamma\left(a_{p}\left(t_{k}\right)+\Delta_{p} \dot{a}_{p}\left(t_{k}\right)\right),
\end{array}
$$

where $\Delta_{p}=50 \mathrm{~ms}$ is acceleration prediction time interval, $\tau_{\circ}$ is the magnitude of assistance torque, $\gamma=10 \mathrm{Ns}^{2}$ is a constant scaling coefficient. Once the pelvis perturbation was detected, assistance torque, $\tau_{\circ}$, was provided after a delay of $55 \mathrm{~ms}$ in the form of a $200 \mathrm{~ms}$ hip flexion torque with a $5 \mathrm{~ms}$ low-pass filter to the leg performing a stepping action (See Fig. 1b) for a recorded pelvis perturbation and hip assistance). The assistance design parameters $\left(a_{\text {lim }}, \Delta_{p}\right.$, $\gamma$ ) were set in pilot testing trials with several subjects such that the torques occurred while the leg was in the air and participants had no complaints about interference from the assistance. Other studies on walking assistance with exoskeletons also used impulse-like assistive torque patterns which, however, were adjusted for each human-subject [2], [18].

\section{EXPERIMENT}

LOPES III robotic exoskeleton. The LOPES III lower limb robotic exoskeleton used in the experiments [10] (Fig. 1a) is composed of leg and pelvis attachments with horizontal push/pull rods connected to robotic shadow legs, an actuation mechanism with electric motors and controllers, and a treadmill. The shadow legs can be actuated in shank flexion/extension, thigh flexion/extension and abduction/adduction. The actuation mechanism enables pelvis control in forward/backward and mediolateral directions. Additionally, LOPES III is equipped with adjustable body weight support for a user. The exoskeleton's passive ankle joints are connected to the leg guidance bars with a series of revolute joints with axes intersecting in the ankle joint. The distances between hip, knee and ankle joints are adjustable for each user to enable natural and comfortable movements. The exoskeleton is operated in admittance control [19] at $1 \mathrm{kHz}$ based on the measured human-exoskeleton interaction forces which are converted into desired exoskeleton joint positions to enable haptic transparency and rendering effects. 
During the experiments LOPES actuators were operated in haptically transparent mode with the pelvis anterior-posterior active degree-of-freedom (DoF) used for force perturbations and right thigh movements (hip joint) used for balance recovery assistance.

Protocol and participants. Four healthy adult participants (1 female, 3 males; age 25-35) took part in the study which was conducted at the University of Twente, Netherlands, and was approved by the institutional ethical committee. In the beginning of the experiment the participants stood upright with their feet together, as shown in Fig. 1a. All participants were right-handed and right-footed, i.e. they preferred to use their right leg for stepping. To ensure that this was the case in all trials, we asked the participants to shift their weight toward the left leg.

A $200 \mathrm{~ms}$ forward perturbation (step filtered with a lowpass filter with $50 \mathrm{~ms}$ time constant) (Fig. 1b) was applied randomly every 4 to $10 \mathrm{~s}$ (uniform distribution), requiring subjects to take a step with the right leg to regain stability. The magnitude of the perturbation was approximately $35 \%$ of body weight (test trials demonstrated that such perturbations require stepping action for balance recovery). This was determined by initialisation trials preceding the experimental procedure to cause loss of postural stability without causing discomfort. The participants were instructed to hold the harness and not use their arms for balance recovery. After each step, subjects returned to an upright stance and stood straight for the next perturbation.

The experiment was composed of three phases: 1) baseline: balance recovery without robotic assistance, 2) assistance: balance recovery with robotic assistance (split into early and late assistance), 3) catch trials: balance recovery without assistance interspersed with assistance trials. Before the experiment the participants familiarised with the system, which took around 2-3 minutes per participant. The goal of the baseline experimental stage was to record balance recovery behavior after pelvis perturbations when the exoskeleton was haptically transparent and no assistance was used. In total 30 pelvis perturbations were introduced in the baseline phase. The baseline phase was followed by an assistance phase of 60 trials during which robotic assistance was applied to support stepping and balance. Six catch trials with robotic assistance removed were randomly introduced during last 30 trials of the assistance phase. The catch trials were used to investigate whether there was any learning in the assistance phase. The overall experiment took around 15-17 minutes per participant.

Data processing. The experimental data recorded at $1 \mathrm{kHz}$ included joint angles and participant-exoskeleton interaction torques for hip and knee flexion/extension; pelvis position and velocity in horizontal plane, and centre of pressure $(\mathrm{CoP})$ measured from the treadmill. Interaction torques were estimated by the exoskeleton's low level real time controller based on the measured human-exoskeleton interaction forces and the segments' lengths [10].

The stepping duration in balance recovery was calculated using the CoP. A threshold was set at $20 \%$ of the maximum lateral centre of pressure velocity in the full duration of the experiment. The end of each recovery step was defined as the time point after the perturbation at which the centre of pressure forward velocity crossed this threshold.

The forward step length was computed as the foot position w.r.t. the initial foot position before perturbation averaged over the $200 \mathrm{~ms}$ following the heel contact. Pelvis position along the anterior-posterior axis was set to zero at the onset of each perturbation, and the right foot $x$ position was calculated using the recorded right hip flexion $\left(\theta_{\mathrm{HF}}\right)$ and knee flexion $\left(\theta_{\mathrm{KF}}\right)$ angles and the estimated leg segment lengths: $x_{\text {foot }}=x_{\text {pelvis }}+l_{\text {thigh }} \sin \theta_{\mathrm{HF}}+l_{\text {shank }} \sin \theta_{\mathrm{KF}}$.

Electromyography (EMG) recordings of muscles on the right leg associated with the hip flexion/extension (GM - Gluteus Maximus, BF- Biceps Femoris, RF - Rectus Femoris, and gastrocnemius) were obtained at $1 \mathrm{kHz}$ and saved during balance recovery with Delsys Trigno wireless EMG system. The rectus femoris muscle is involved in hip flexion, while GM and BF muscles contribute to hip extension. RF and $\mathrm{BF}$ are biarticular muscles that have been shown to have non-intuitive function depending on configuration [20], [21]. Additionally, the gastrocnemius muscle activation was recorded to observe any influence of the assistance controller to ankle-foot plantar flexion and knee flexion.

Raw EMG signals were de-meaned, high-pass filtered at $50 \mathrm{~Hz}$, rectified, and low-pass filtered at $10 \mathrm{~Hz}$ (4th order Butterworth). Signals were normalised by subject, such that the highest peak value was $100 \%$ for each leg over all trials. This allowed for comparison between subjects.

Within-subjects and between-subjects statistics were similarly computed for the standing experiment, with a oneway ANOVA comparing baseline, early assistance, late assistance, and catch trials. Because of the smaller number of perturbations in each condition, early assistance consisted of the first 15 trials of the condition, while baseline and late assistance were the last 15 trials of their respective conditions.

Outliers were removed prior to analysis, based on the right hip flexion/extension data within each experimental state. Specific trials with hip trajectories markedly different from the mean over all trials were considered as outliers and eliminated. This was detected from the root-mean squared error (RMSE) relative to the overall mean, with a threshold of two times the average RSME. Furthermore, trials in which the peaks of at least one of the EMG recordings exceeded the double standard deviation margins around the mean recorded values were also eliminated from the analysis. This was done to exclude the trials with abstruse EMG recordings which could be caused by body movement artifacts. It was verified that no more than $10 \%$ of trials per experimental stage were removed for each participant.

\section{RESUlTS}

We analysed the right leg movements, muscle activation, pelvis and centre of pressure kinematics in stepping during balance recovery for each participant per experimental phase. The results per participant and experimental phase are 


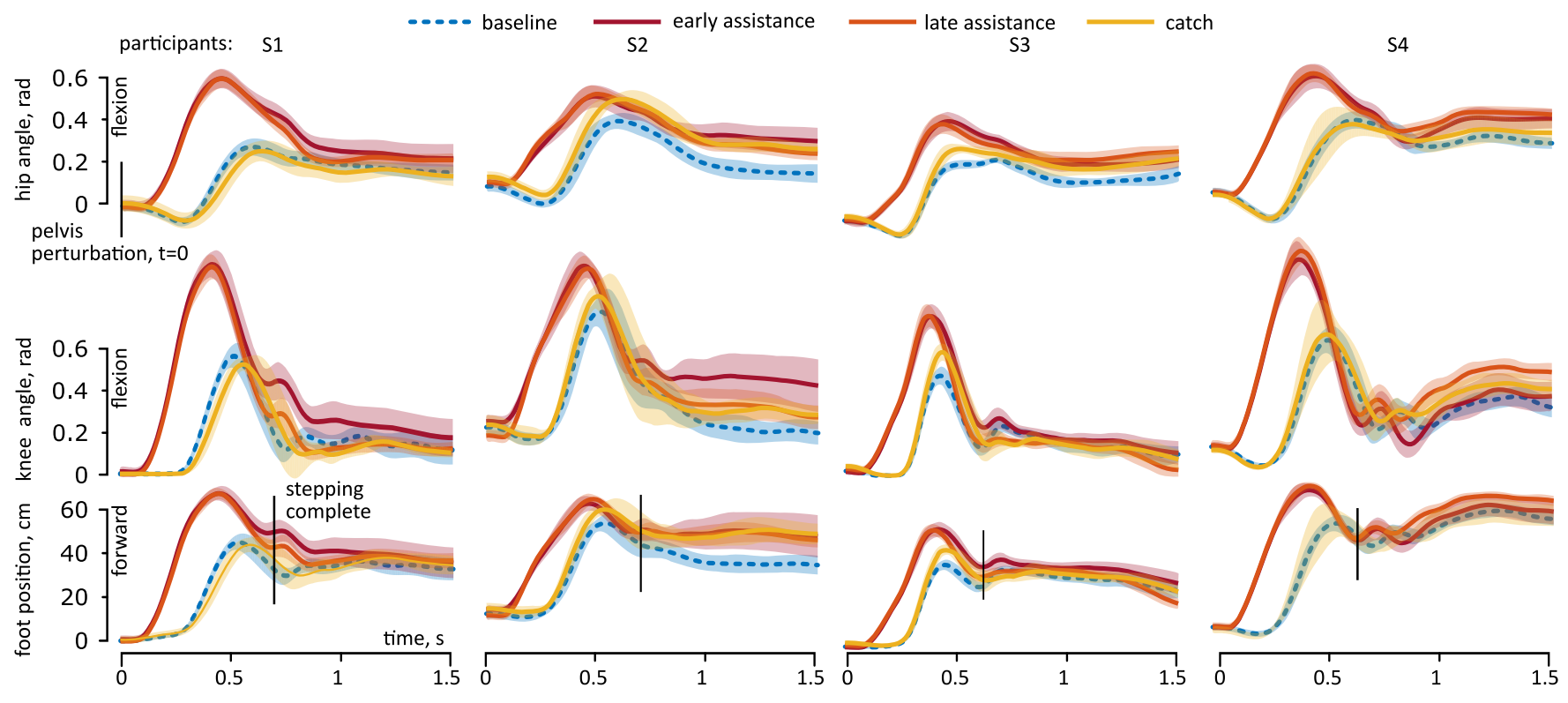

Fig. 2. Right leg kinematic data during stepping for the four participants (S1-S4). Black vertical line in the last row corresponds to completion of stepping.

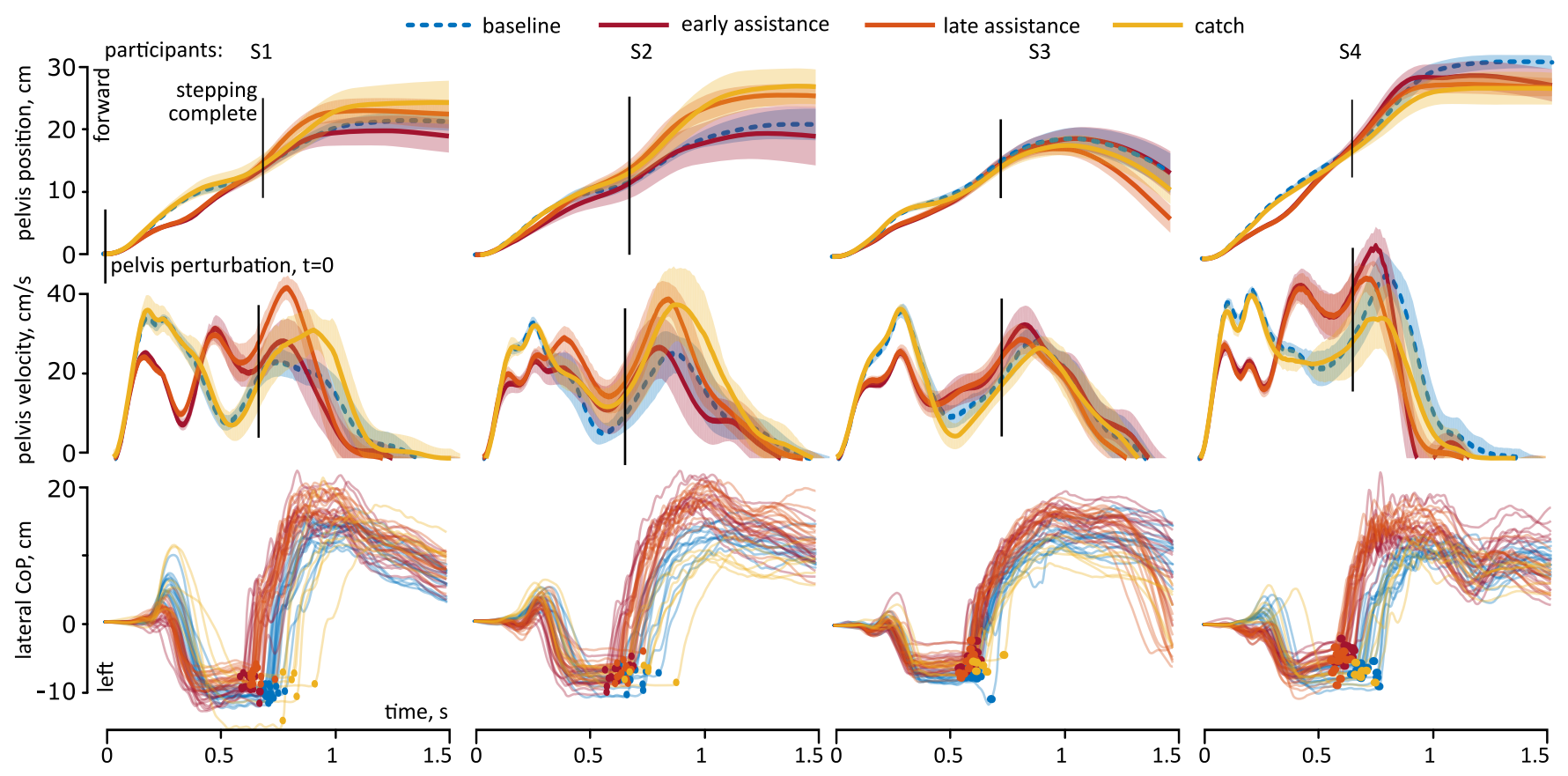

Fig. 3. Pelvis AP position, velocity and lateral centre of pressure during stepping for all participants (S1-S4).

presented as follows: right leg kinematics in Fig. 2, pelvis position, velocity and centre of pressure in Fig. 3, right leg muscles' activation in Fig. 4. In these figures the recordings are aligned in time, such that the time instant $t=0$ corresponds to the moment when a forward force perturbation was applied. We have also computed the within-participant and the between- participant statistical significance in the data, as shown in Table I and Fig. 5, respectively.

Hip flexion. The first row of Fig. 2 shows the mean (bold lines) and standard deviation margins (in shadow) for the right hip flexion recordings in four experimental stages (shown in four colours) for each participant (columns S1-S4).
Once a pelvis perturbation was applied a participant had to flex his/her right hip in order to move the right foot forward and recover balance with stepping. Hence, in all participants we see an increase of hip flexion during approximately the first $0.5 \mathrm{~s}$. Stepping was complete at approximately $0.7 \mathrm{~s}$, when the right foot was placed on the ground and hip angle was static. For all participants a clear difference in trajectories between baseline and assistance is observed. The recordings in catch trials are close to the baseline trajectories. Assistance torque applied to the hip during the assistance trials increased the hip flexion magnitude compared to the baseline cases. Hip extension movement which is observed 
TABLE I

WITHIN-PARTICIPANT STATISTICAL ANALYSIS (T-TEST, SIGNIFICANCE

\begin{tabular}{|c|c|c|c|c|}
\hline \multirow{2}{*}{ Variable } & \multirow{2}{*}{$\begin{array}{l}\text { FOR } p<0.05) \\
\text { Experimental stage }\end{array}$} & \multicolumn{3}{|c|}{$\begin{array}{c}\text { \# of significant } \\
\text { participants }\end{array}$} \\
\hline & & $<$ & $=$ & $>$ \\
\hline \multirow[t]{5}{*}{ mean hip flexion } & Baseline vs. Early & 4 & 0 & 0 \\
\hline & Baseline vs. Late & 4 & 0 & 0 \\
\hline & Catch vs. Early & 4 & 0 & 0 \\
\hline & Catch vs. Late & 4 & 0 & 0 \\
\hline & Early vs. Late & 0 & 4 & 0 \\
\hline \multirow[t]{5}{*}{ mean knee flexion } & Baseline vs. Early & 4 & 0 & 0 \\
\hline & Baseline vs. Late & 4 & 0 & 0 \\
\hline & Catch vs. Early & 4 & 0 & 0 \\
\hline & Catch vs. Late & 4 & 0 & 0 \\
\hline & Early vs. Late & 1 & 1 & 2 \\
\hline \multirow[t]{4}{*}{ step duration } & Baseline vs. Early & 0 & 1 & 3 \\
\hline & Catch vs. Early & 0 & 0 & 4 \\
\hline & Catch vs. Late & 0 & 0 & 4 \\
\hline & Early vs. Late & 0 & 4 & 0 \\
\hline \multirow[t]{3}{*}{ step length } & Baseline vs. Early & 3 & 1 & 0 \\
\hline & Baseline vs. Late & 2 & 2 & 0 \\
\hline & Baseline vs. Catch & 0 & 4 & 0 \\
\hline \multirow[t]{6}{*}{ pelvis velocity } & Baseline vs. Early & 0 & 0 & 4 \\
\hline & Baseline vs. Late & 0 & 0 & 4 \\
\hline & Baseline vs. Catch & 0 & 4 & 0 \\
\hline & Catch vs. Early & 0 & 0 & 4 \\
\hline & Catch vs. Late & 0 & 0 & 4 \\
\hline & Early vs. Late & 0 & 4 & 0 \\
\hline stability & Baseline vs. Early & 3 & 1 & 0 \\
\hline \multirow[t]{4}{*}{ metric } & Baseline vs. Late & 3 & 1 & 0 \\
\hline & Baseline vs. Catch & 0 & 4 & 0 \\
\hline & Catch vs. Early & 3 & 1 & 0 \\
\hline & Catch vs. Late & 3 & 1 & 0 \\
\hline
\end{tabular}

during first 200-300 ms after the perturbation in the baseline trials is not present in early and late assistance trials. Hip flexion in catch trials is similar to the baseline trials.

Within participant analysis showed that for all participants the mean hip flexion with assistance was significantly larger than in baseline or catch trials (Table I). For all participants, no statistical difference was observed between baseline and catch trials. Between participant analysis showed larger hip flexion in early and late assistance than in baseline trials, which was statistically significant $(p<0.05)$, as shown in Fig. 5a. There was no significant difference between baseline and catch trials. There was also no significant difference in hip flexion between early and late assistance trials in all participants.

Knee flexion Right knee flexion recordings for each participant are shown in the second row of Fig. 2. Similar to hip movements, we observe knee flexion during $0.5 \mathrm{~s}$ after the perturbation, after which it extends to approximately 0.3 $0.4 \mathrm{rad}$ when the stepping is complete (even though there was no direct exoskeleton torque assistance applied at the knee level). Trials with assistance exhibited larger knee angular displacements than in the baseline and the catch stages of the experiment. Knee flexion in the catch and the baseline trials do not differ from each other significantly.

Similar to hip flexion, within participant analysis showed that for all participants the mean knee flexion was significantly increased in assistance trials compared to baseline and catch trials (Table I). For three subjects there was no difference in knee flexion in baseline and catch trials Results of between participant analysis of knee flexion are shown in Fig. 5b. It showed that knee flexion was increased significantly in all assistance trials $(p<0.05)$.

Foot placement, step length and duration. Calculated trajectories for right foot position in the forward direction are shown in the last row of Fig. 2. During the assistance trials, the right foot was placed further away in conjunction with increased hip flexion and knee extension when compared to baseline and catch trials, which is clearly observed for the participants S1, S2 and S4. Within-participant analysis showed that the step length was increased in the early assistance trials compared to the baseline recordings for these three participants. For all participants, there was no significant difference between the baseline and catch trials (See Table I). Between participant analysis revealed no significant differences in step length between experimental stages $(p=0.15, F=2.14)$.

Within participant analysis showed that step duration was longer in catch trials than in early and late assistance for all participants (Table I). There was no significant difference in step duration between early and late assistance trials. Three participants had reduced step duration in early assistance compared to baseline trials $(p<0.05)$. Between-participant analysis showed that step duration was significantly increased in catch trials compared to assistance trials $(p<0.05)$, as presented in Fig. 5.

Pelvis movement. Pelvis forward movement trajectories, velocity and CoP lateral displacement are shown in Fig. 3. The pelvis moved forward once the force perturbation was applied until the stepping for balance recovery was complete, with the body slowing and stopping at $15-30 \mathrm{~cm}$ distance from the origin. As seen from the first row in Fig. 3, the recordings of pelvis position during stepping are characterised with small standard deviation for participants S1, S3 and S4. For the same participants, the pelvis movement forward in the assistance trials was slowed down from around $200 \mathrm{~ms}$ when right hip assistance torque was applied. This can be clearly seen in the second row of Fig. 3: the pelvis velocity is significantly lower in the early and late assistance cases compared to baseline and catch trials during the perturbation and assistance actions (approx. first $400 \mathrm{~ms}$ ), for all subjects. Probably, slower pelvis movements were the result of the reaction torques caused by fast hip flexion movements. We analysed the statistical significance for the pelvis velocity at time $\mathrm{t}=200 \mathrm{~ms}$ when the pelvis perturbation was complete but the hip flexion assistance was still active. Between participant ANOVA analysis revealed significant differences in pelvis velocity between experimental stages for all participants $(p<0.001, F=48.37$, Fig. 5d). As shown in Table I for the within-participant analysis of all subjects, pelvis velocity was decreased at the end of the perturbation as a result of stepping assistance $(p<0.001)$.

Stability. One of the ways to analyse stability of recovery relevant to our experiment is to take into account the distance between the stepping foot and the centre of mass $(\mathrm{CoM})$ after the stepping is complete. Such measure allows quantitative 


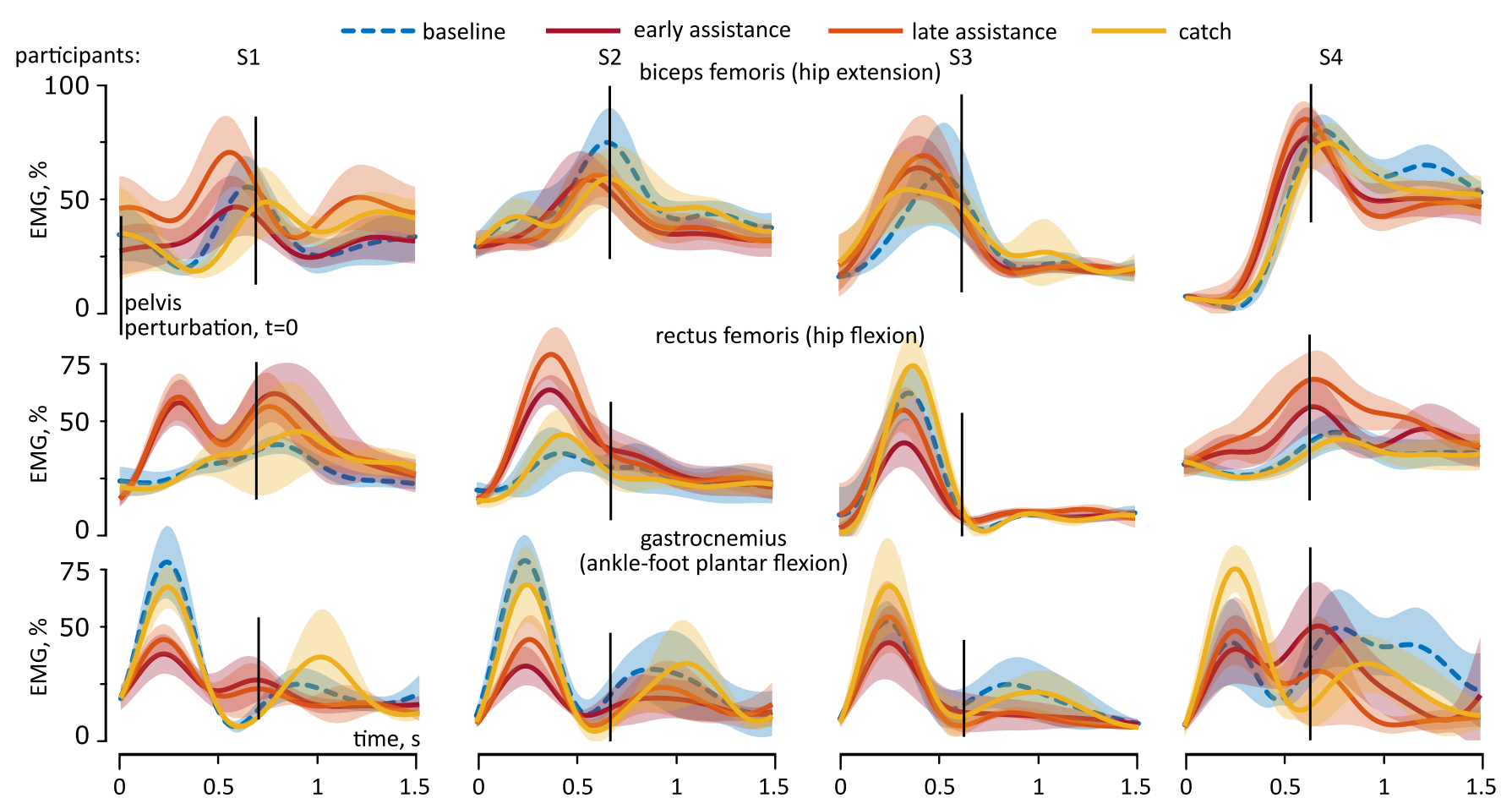

Fig. 4. Normalised right leg muscle activity recordings during stepping for all participants (S1-S4).

comparison of postural stability in assisted and non-assisted trials and is a simplified version of the stability margin introduced in [22]. One will prevent falling over if the stepping foot is placed more anteriorly than the centre of mass. In our case the average distance in $\mathrm{cm}$ between the pelvis position (assumed to be close to CoM) and the right foot after the stepping was approximately $19 \pm 5,27 \pm 6,25 \pm 7$ and $20 \pm 7$ (mean \pm sd) for baseline, early and late assistance, and catch trials, respectively. This result demonstrates that the stability of standing was improved during exoskeletonassisted trials and it was statistically significant for three subjects as shown in Table I.

Lateral CoP trajectories with markers indicating the end of stepping are shown in the last row of Fig. 3. Once the perturbation was applied and the right leg was lifted from the ground for balance recovery, the CoP quickly deviated to the left until the stepping was complete. Once stable double stance was achieved, the CoP moved to the right as the overall CoM of the body was deviated to the right as well. For all participants except for S2, it can be clearly seen that stepping was complete faster compared to baseline and catch trials (see the third row of Fig. 3). Also, the CoP lateral deviation to the left in the assistance trials was slightly smaller for participants S1, S3 and S4.

Muscle activity. The normalised muscle activation of the right leg during stepping is presented in Fig. 4. There was no difference in integrated BF EMG (first row of Fig. 4) between conditions in the between-participants ANOVA ( $p=0.55$, $F=0.74)$. However, within-participant analysis showed that three subjects had increased BF muscle contraction from baseline to late assistance, and for all participants there was no significant difference between the muscle activation in baseline and catch trials.

The second row of Fig. 4 shows activation of RF muscle. There were no significant between-participant effects of experimental stage on integrated RF contraction ( $p=0.11$, $F=2.49$ ). However, within-participant analysis showed that three participants had increased RF contraction in assistance trials compared to baseline and catch trials; for all participants there was no difference of activation in baseline and catch trials; three participants showed an increase from early to late assistance. The last row of Fig. 4 shows activation of gastrocnemius muscle. There was no main effect of experimental stage on gastrocnemius contraction in the between-subject analysis.

\section{Discussion}

Previous studies [13], [15], [14], [16] have investigated some aspects of balance with a robotic exoskeleton using testing with a single subject and the study [17] investigated assisted balance recovery following slippage in walking with eight elderly participants. In this context, this paper has presented the first study which systematically analyses balance recovery in standing through stepping with a robotic exoskeleton on four subjects. Compared with [17], in our study in addition to kinematic responses we measured and analysed muscle activation. The results demonstrated that a simple controller can be efficiently used for assisting balance in complex physical interactions between a user and robotic exoskeleton. We could analyse important behavioral trends, although some observations are not statistically significant due to the complexity of the experimental setup used in this 
work and the variability of human motor commands. The results can be analyzed with respect to the three predictions $\mathrm{H}_{1}-\mathrm{H}_{3}$ described in the Introduction.

Adaptation to assistance. First, the results showed that the participants could easily adapt to the robotic assistance, which did require learning and did not affect the natural stepping response. This is important as assistance should not alter the healthy behavior. A statistical comparison of early and late assistance trials did not reveal any differences and balance recovery performance remained same during the assisted trials. A user's neuromuscular motor control could rapidly adapt to the forces and movements produced by the robot and no motor skills learning was required. There was no statistically significant difference in balance recovery between non-assisted baseline and catch trials demonstrating that the participants were able to return to their typical balance response once robotic assistance was disabled.

Kinematic response. The experimental results also verified our second prediction $\left(\mathrm{H}_{2}\right)$ that the exoskeleton could speed up the stepping. Balance recovery assistance modified the stepping leg trajectories by increasing hip and knee movement and it increased the reaction speed and decreased the step duration. Similarly to [17] the assistance resulted in a significant change of the movement kinematics. The hip flexion assistance torque resulted in greater average hip flexion during the step. Notably, this appears to be due to the hip flexion occurring earlier, rather than simply causing a larger peak flexion due to the presence of external assistance torques. This is because the assistance torque was applied $55 \mathrm{~ms}$ following the detection of the perturbation, as opposed to the reaction delay of up to $100-200 \mathrm{~ms}$ for the subjects' muscle activation which is similar to the results reported in [7], [8], [9], [23]. In our case we achieved faster balance

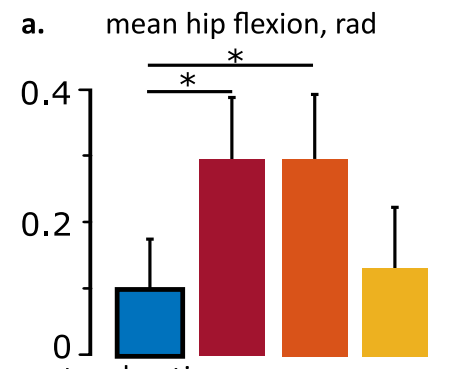

c. step duration, $\mathrm{s}$

d. pelvis velocity, $\mathrm{cm} / \mathrm{s}$
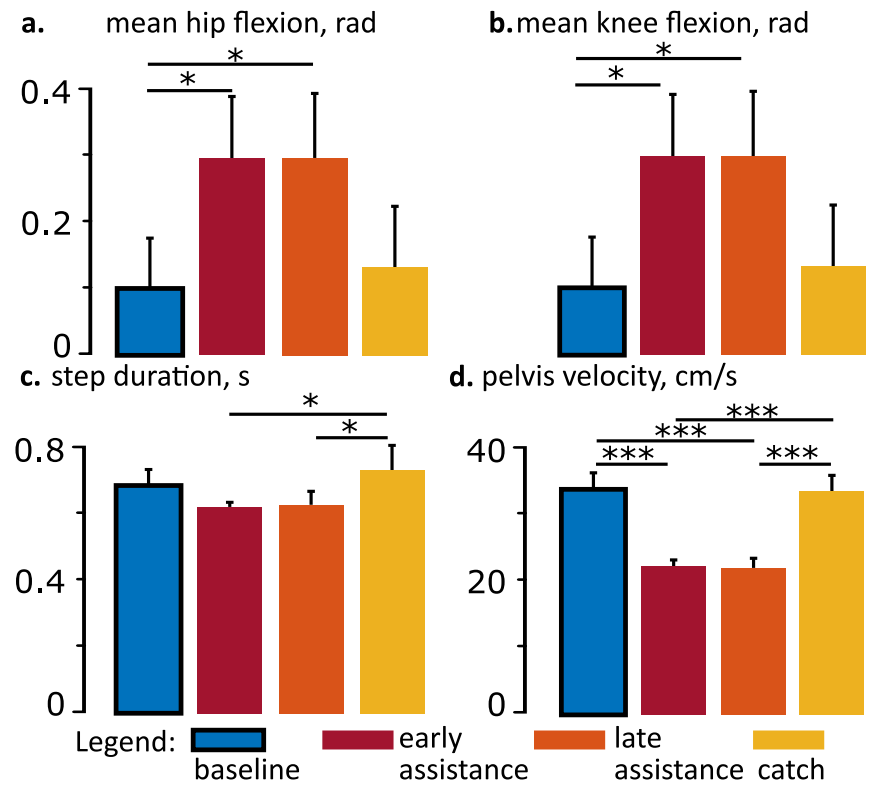

Fig. 5. Results of the between-participant statistical analysis for right leg stepping: a-c - mean kinematic parameters for all participants; d - pelvis $\mathrm{AP}$ velocity at the end of perturbation. recovery by applying the assistance earlier than the subjects motor response onset times. However, as discussed later the timing of assistance should be taken into account to prevent increased muscle activation. Interestingly, a similar increase in knee flexion was observed in assistance trials even though the robotic assistance was applied only to hip movements. This indicates that it is possible to apply robotic assistance only to selected DoFs of the human body and the (rest of the) body will naturally adjust balance recovery response through neuromechanical interaction.

Postural stability was improved as a result of faster and larger joint movements because the foot placement with respect to the pelvis (CoM) was increased in assisted trials providing better support base. The observed larger step size in combination with a faster swing time assure that the CoM can be more effectively decelerated and so the perturbation counteracted. We see this as a positive result, as the study was conducted with healthy subjects who can already take appropriately sized steps to recover balance, and the assistance provided did not negatively impact this ability. Steps duration was slightly longer without assistance (catch and baseline trials) than in assisted trials, indicating that subjects adapted their behavior in anticipation of the assistance, and completing the recovery step took longer when the expected assistance was absent.

Muscle activation. The third prediction $\left(\mathrm{H}_{3}\right)$ was not verified, as muscle activation was increased when assistance was provided by the exoskeleton. The analysis showed that muscular contraction in RF and BF (hip flexion/extension movement) often increased as a result of assistance. However, there was no significant difference in the betweenparticipants analysis likely due to high between-subject variability. The within-subjects differences underlined the importance of selecting appropriate assistance torque magnitudes and timing parameters. Interestingly, the betweenparticipants analysis also showed a trend for reduced gastrocnemius muscle activity (ankle-foot plantar flexion movement) indicating that less ankle activity is required to push off for stepping. Even though this decrease was statistically insignificant, it demonstrates that the participants' whole body neuromuscular control was actively adapting to exoskeleton assistance forces. The GM muscle does not appear to have been significantly involved in the movement or impacted by the assistance.

Overall, the effect of assistance on muscular activation is mixed. While the GM appears to not be involved in or affected by the assistance, activation of BF and RF (both muscles in the thigh) apparently increased, and gastrocnemius muscle contraction decreased. The gastrocnemius is the only muscle that showed a reduction of contraction and is also the only muscle measured that is not responsible for hip movement. The RF and BF are antagonistic muscles [20], [21], but both showed a tendency toward increasing contraction; added assistance led to increased stiffening through co-contraction of the muscles responsible for hip flexion and extension. We think that the main reason for the increased muscle activity in the assisted trials is the difference in time 
when assistance is applied by the exoskeleton and when the motor commands activate the hip flexion muscles as a response to the perturbation. It appeared that the robot was acting faster which led to a certain level of resistance from the users body at earlier stages of balance recovery. This issue can be addressed by carefully adjusting the assistance's timing and it is also related to the time instant when the perturbation is detected (pelvis acceleration threshold). One approach could be to adjust the assistance timing to each user with the exoskeleton support optimisation techniques that were already used to tune walking assistance [18].

The limitations of our study include the limited number of participants and the experimental scenario which only considered forward perturbations in standing. Compared with the experiment in [17] the exoskeleton used in the study was partially grounded (the electrical actuators of the LOPES system are attached to a rigid grounded frame), however, stiff and low-friction mechanism with high-performance admittance controller enabled us to realistically emulate a mobile wearable lower limb exoskeleton.

Adaptation for impaired users. The presented assistance method could be adapted for impaired users. In this case, the exoskeleton first should be actively supporting the impaired muscle function of the lower limbs all the time (at ankle, knee, hip and pelvis joints). At this time there are no published studies on full lower limb exoskeleton based balance assistance for impaired users. Only two studies report on partial assistance: at the foot-ankle level for people with spinal cord injury [16] and at the hip level for elderly and transfemoral amputees [17]. In general impairment case to enable full lower limb support in balancing the exoskeleton's controller should have or acquire knowledge of which joints should be supported during the stepping action (e.g. left or right hip). Recent applications have demonstrated that using exoskeletons to support impaired users requires the development of user-specific biomechanical modeling which can be integrated into the walking assistance controllers [2], [24], [25].

\section{ACKNOWLEDGMENT}

This research was supported by the EU FP7 ICT-601003 BALANCE and ICT-611626 SYMBITRON grants. J. Ebert was also funded the U.K. Marshall Aid Commemoration Commission.

\section{REFERENCES}

[1] P. Beyl, M. Van Damme, R. Van Ham, B. Vanderborght, and D. Lefeber, "Design and control of a lower limb exoskeleton for robotassisted gait training," Appl Bionics Biomech, vol. 6(2), 2009.

[2] S. A. Murray, K. H. Ha, C. Hartigan, and M. Goldfarb, "An assistive control approach for a lower-limb exoskeleton to facilitate recovery of walking following stroke," IEEE Trans Neural Syst Rehabil Eng, vol. 23, no. 3, pp. 441-449, 2015.

[3] M. Bortole, A. Venkatakrishnan, F. Zhu, J. C. Moreno, G. E. Francisco, J. L. Pons, and J. L. Contreras-Vidal, "The h2 robotic exoskeleton for gait rehabilitation after stroke: early findings from a clinical study," $J$ Neuroeng Rehabil, vol. 12, no. 1, p. 54, 2015.

[4] G. Aguirre-Ollinger, J. E. Colgate, M. A. Peshkin, and A. Goswami, "Active-impedance control of a lower-limb assistive exoskeleton," in 10th IEEE Int Conf on Rehabilitation Robotics, 2007, pp. 188-195.
[5] L. Wang, S. Wang, E. H. van Asseldonk, and H. van der Kooij, "Actively controlled lateral gait assistance in a lower limb exoskeleton," in IEEE/RSJ Int Conf on Intelligent Robots and Systems, 2013.

[6] H. van der Kooij, E. H. van Asseldonk, and M. Vlutters, "Towards exoskeletons with balance capacities," in Wearable Robotics: Challenges and Trends. Springer, 2017, pp. 175-179.

[7] M. Do, Y. Breniere, and S. Bouisset, "Compensatory reactions in forward fall: are they initiated by stretch receptors?" Electroencephalography and clinical Neurophysiology, vol. 69, no. 5, 1988.

[8] R. Peterka and F. Black, "Age-related changes in human posture control: motor coordination tests," J Vestib Res, vol. 1(1), 1990.

[9] D. Thelen, M. Muriuki, J. James, A. Schultz, J. Ashton-Miller, and N. Alexander, "Muscle activities used by young and old adults when stepping to regain balance during a forward fall," Journal of Electromyography and Kinesiology, vol. 10, no. 2, pp. 93-101, 2000.

[10] J. Meuleman, E. van Asseldonk, G. van Oort, H. Rietman, and $H$. van der Kooij, "Lopes iidesign and evaluation of an admittance controlled gait training robot with shadow-leg approach," IEEE Trans Neural Syst Rehabil Eng, vol. 24, no. 3, pp. 352-363, 2016.

[11] C. Doppmann, B. Ugurlu, M. Hamaya, T. Teramae, T. Noda, and J. Morimoto, "Towards balance recovery control for lower body exoskeleton robots with variable stiffness actuators: Spring-loaded flywheel model," in IEEE Int Conf on Robotics and Automation, 2015.

[12] B. Ugurlu, C. Doppmann, M. Hamaya, P. Forni, T. Teramae, T. Noda, and J. Morimoto, "Variable ankle stiffness improves balance control: Experiments on a bipedal exoskeleton," IEEE/ASME T on mechatronics, vol. 21, no. 1, pp. 79-87, 2016.

[13] D. Martelli, F. Vannetti, M. Cortese, P. Tropea, F. Giovacchini, S. Micera, V. Monaco, and N. Vitiello, "The effects on biomechanics of walking and balance recovery in a novel pelvis exoskeleton during zero-torque control," Robotica, vol. 32, no. 8, pp. 1317-1330, 2014.

[14] P. Stegall, K. Winfree, D. Zanotto, and S. K. Agrawal, "Rehabilitation exoskeleton design: Exploring the effect of the anterior lunge degree of freedom," IEEE Trans on Robotics, vol. 29, no. 4, 2013.

[15] N. Karavas, A. Ajoudani, N. Tsagarakis, and D. Caldwell, "Humaninspired balancing assistance: Application to a knee exoskeleton," in IEEE Int conf on robotics and biomimetics, 2013, pp. 292-297.

[16] A. Emmens, I. Pisotta, M. Masciullo, E. van Asseldonk, and H. van der Kooij, "Improving the standing balance of people with spinal cord injury through the use of a powered ankle-foot orthosis," in Wearable Robotics: Challenges and Trends. Springer, 2017, pp. 415-419.

[17] V. Monaco, P. Tropea, F. Aprigliano, D. Martelli, A. Parri, M. Cortese, R. Molino-Lova, N. Vitiello, and S. Micera, "An ecologicallycontrolled exoskeleton can improve balance recovery after slippage," Scientific reports, vol. 7, p. 46721, 2017.

[18] Y. Ding, M. Kim, S. Kuindersma, and C. J. Walsh, "Human-in-theloop optimization of hip assistance with a soft exosuit during walking," Science Robotics, vol. 3, no. 15, p. eaar5438, 2018.

[19] R. Q. Van der Linde, P. Lammertse, E. Frederiksen, and B. Ruiter, "The hapticmaster, a new high-performance haptic interface," in Proc. Eurohaptics, 2002, pp. 1-5.

[20] A. S. Arnold, F. C. Anderson, M. G. Pandy, and S. L. Delp, "Muscular contributions to hip and knee extension during the single limb stance phase of normal gait: a framework for investigating the causes of crouch gait," J Biomech, vol. 38, no. 11, pp. 2181-2189, 2005.

[21] A. Hernández, Y. Dhaher, and D. G. Thelen, "In vivo measurement of dynamic rectus femoris function at postures representative of early swing phase," J Biomech, vol. 41, no. 1, pp. 137-144, 2008.

[22] A. Hof, M. Gazendam, and W. Sinke, "The condition for dynamic stability," J Biomech, vol. 38, no. 1, 2005.

[23] M. Vlutters, E. H. van Asseldonk, and H. van der Kooij, "Lower extremity joint-level responses to pelvis perturbation during human walking," Scientific reports, vol. 8, no. 1, 2018.

[24] A. R. Wu, F. Dzeladini, T. J. Brug, F. Tamburella, N. L. Tagliamonte, E. H. Van Asseldonk, H. Van Der Kooij, and A. J. Ijspeert, "An adaptive neuromuscular controller for assistive lower-limb exoskeletons: A preliminary study on subjects with spinal cord injury," Frontiers in neurorobotics, vol. 11, 2017.

[25] H.-Y. Huang, I. Farkhatdinov, A. Arami, and E. Burdet, "Modelling neuromuscular function of sci patients in balancing," in Converging Clinical and Engineering Research on Neurorehabilitation II. Springer, 2017. 